\title{
Variation in haematological parameters in children less than five years of age with asymptomatic Plasmodium infection: implication for malaria field studies
}

\author{
Adama Gansane ${ }^{1 /+}$, Issa Nebie Ouedraogo' ${ }^{1}$ Noelie Bere Henry', Issiaka Soulama', \\ Esperance Ouedraogo', Jean-Baptiste Yaro', Amidou Diarra1, Sombie Benjamin', \\ Amadou Tidiani Konate ${ }^{1}$, Alfred Tiono', Sodiomon Bienvenu Sirima ${ }^{1,2}$ \\ ${ }^{1}$ Centre National de Recherche et de Formation sur le Paludisme, Kadiogo, Ouagadougou, Burkina Faso \\ ${ }^{2}$ Groupe de Recherche Action en Santé, Kadiogo, Ouagadougou, Burkina Faso
}

\begin{abstract}
During the season of high malaria transmission, most children are infected by Plasmodium, which targets red blood cells (RBCs), affecting haematological parameters. To describe these variations, we examined the haematological profiles of two groups of children living in a malaria-endemic area. A cross-sectional survey was conducted at the peak of the malaria transmission season in a rural area of Burkina Faso. After informed consent and clinical examination, blood samples were obtained from the participants for malaria diagnosis and a full blood count. Of the 414 children included in the analysis, 192 were not infected with Plasmodium, whereas 222 were asymptomatic carriers of Plasmodium infection. The mean age of the infected children was 41.8 months (range of 26.4-57.2) compared to 38.8 months (range of 22.4-55.2) for the control group $(p=0.06)$. The asymptomatic infected children tended to have a significantly lower mean haemoglobin level (10.8 g/dL vs. $10.4 \mathrm{~g} / \mathrm{dL} ; p<0.001)$, mean lymphocyte count


$\left(4.388 \times 10^{6} / \mu \mathrm{L} v \mathrm{v} .4 .158 \times 10^{6} / \mu \mathrm{L} ; p<0.001\right)$ and a higher mean monocyte count $(1403 / \mu \mathrm{L} v \mathrm{~s} .1192 / \mu \mathrm{L} ; p<0.001)$ compared to the control group. Special attention should be applied when interpreting haematological parameters and evaluating immune responses in asymptomatic infected children living in malaria-endemic areas and enrolled in vaccine trials.
\end{abstract}

Key words: Plasmodium - asymptomatic infection - haematology - children - vaccine - Burkina Faso

Malaria remains a major public health problem in Africa (Snow et al. 1999, Rowe et al. 2006). Indeed, in Burkina Faso, malaria is the most common cause of hospitalisation and death, particularly for children under five years of age (DEP/SNIS 2007).

As malaria parasites target red blood cells (RBCs), haematological changes are one of the most common complications and thus play a major role in the outcomes of the disease. Several blood constituents, including RBCs, platelets and leucocytes, are affected during malaria and serious anaemia is the most common complication in children with severe malaria in the rural areas of Burkina Faso and other parts of Africa (Stein \& Gelfand 1985, Amodu et al. 1998, Richards et al. 1998, Schellenberg et al. 1999).

A study conducted in American soldiers reported that leucocytosis was more frequent in Plasmodium vivax-infected African Americans compared to Caucasians, whereas lymphocytosis was more common in the

doi: $10.1590 / 0074-0276108052013017$

Financial support: NIH/NIAID/DMID (DMID 06-20/HH-

SN266200400016C)

+ Corresponding author: agansane.cnrfp@fasonet.bf

Received 24 May 2012

Accepted 16 May 2013 latter (Fisher et al. 1970). Perera et al. (1994) showed that malaria-naïve Sri Lankans infected with $P$. vivax had a higher mean lymphocyte count in comparison to malariaimmune $P$. vivax-infected patients. In a study conducted in Thailand, people with patent parasitaemia tended to have significantly lower white blood cell (WBC), RBC, platelet and haemoglobin ( $\mathrm{Hb}$ ) levels than individuals who were malaria negative (Erhart et al. 2004). In their studies, Ladhani et al. (2002) and Kassa et al. (2006) concluded that malaria can significantly reduce the number of total and differential circulating WBCs. In Kenya, Maina et al. (2010) showed that platelet, lymphocyte, eosinophil and RBC counts and $\mathrm{Hb}$ levels were lower in malaria-infected children, whereas absolute monocyte and neutrophil counts and the mean platelet volume were higher in comparison to uninfected children.

The different studies cited above emphasise the wellknown haematological variations for severe malaria and acute malaria, particularly in adults, and for parameters, such as $\mathrm{Hb}$ or packed cell volume used to evaluate anaemia and WBCs. Although there is evidence that malaria can affect the profile of circulating cells in peripheral blood, this finding might not be consistent among all geographical locations. Indeed, haematological changes related to malaria are subject to variation depending on the level of disease endemicity, nutritional status, genetic factors, sociodemographic conditions, ethnicity and immunity (Hill et al. 1991). Moreover, few studies have been conducted to explore the haematological changes 
in children with the asymptomatic carriage of malaria parasites and living in malaria-endemic areas in the western part of Africa.

In malaria-endemic areas where Phases $\mathrm{Ib}, \mathrm{II}$ and III vaccine trials are usually implemented, nearly all the children during the season of high malaria transmission are infected with Plasmodium, a parasite that targets RBCs and can affect WBCs. Moreover, vaccine efficacy based on cellular responses and correlated with clinical protection is evaluated using WBCs and subsets and the methods used require a high level of viable peripheral blood mononuclear cells (PBMCs) after blood collection. Thus, the impact of malaria parasites on WBCs could affect the validity of in vitro proliferative assays, such as the $\left[{ }^{3} \mathrm{H}\right]$ thymidine uptake assay, which is widely used in areas of endemicity to detect specific cellular responses to Plasmodium falciparum antigens. To determine the potential of these variations as confounding factors at a future malaria vaccine trial site, we examined the haematological profiles of children less than five years of age with asymptomatic Plasmodium infection and living in an area of Burkina Faso in which malaria is endemic.

\section{PATIENTS, MATERIALS AND METHODS}

The haematological study presented here was part of a large epidemiological study conducted to evaluate morbidity and mortality related to malaria.

Study area and patients - This study was conducted in the Sapone Health District and involved a demographic surveillance system (DSS) with a population of 99,895 inhabitants. The study site was located $50 \mathrm{~km}$ southwest of Ouagadougou, the capital city of Burkina Faso. Malaria transmission is hyperendemic and markedly seasonal: it is high during the rainy season, from June-October and low from November-May.

At the peak of the high malaria transmission season, in September 2007, a cross-sectional survey was conduct- ed in a sample of the population of four randomly selected villages in the DSS area of the Sapone Health District: Dawelgue, Tanghin, Kounda and Watenga (Fig. 1). The inclusion criteria were as follows: (i) written informed consent obtained from the parents or legal guardians of the children, (ii) ages of less than five years and (iii) residency in the DSS of Sapone for at least three months. Children with acute or chronic disease were excluded from the study. The sample was identified from a census list generated from the DSS census list of all children under five years of age living in the study region. The medical history of the children during the survey of eligibility criteria fulfilment was recorded, their axillary temperature was measured, a physical examination was conducted and blood samples were obtained for blood films, a complete blood count and a differential blood cell count.

A malaria rapid diagnostic test, OptiMAL ${ }^{\circledR}$ (DiaMed, Cressier, Switzerland), was performed for children with an axillary temperature $\geq 37.5^{\circ} \mathrm{C}$ only for diagnostic purposes and as a routine activity. Sick children were excluded from the study and referred to the closest health centre for prompt and adequate treatment according to the national health guidelines and free of charge.

Blood sample collection and processing - During the cross-sectional survey, blood sample tubes were transported daily to the haematology laboratory of Centre National de Recherche et de Formation sur le Paludisme within 1 $\mathrm{h}$ of collection in cold boxes, with the temperature monitored and maintained between $2-8^{\circ} \mathrm{C}$. A full blood count was then performed using a semi-automated haematology analyser (Pentra 60, ABX, Montpelier, France), which, at the time, was used in the College of American Pathologists proficiency testing programme. Before each series of blood sample tests, quality control was performed and recorded using commercial specimens. In the case of failure, actions were taken in accordance with laboratory procedure and the manufacturer's instructions.
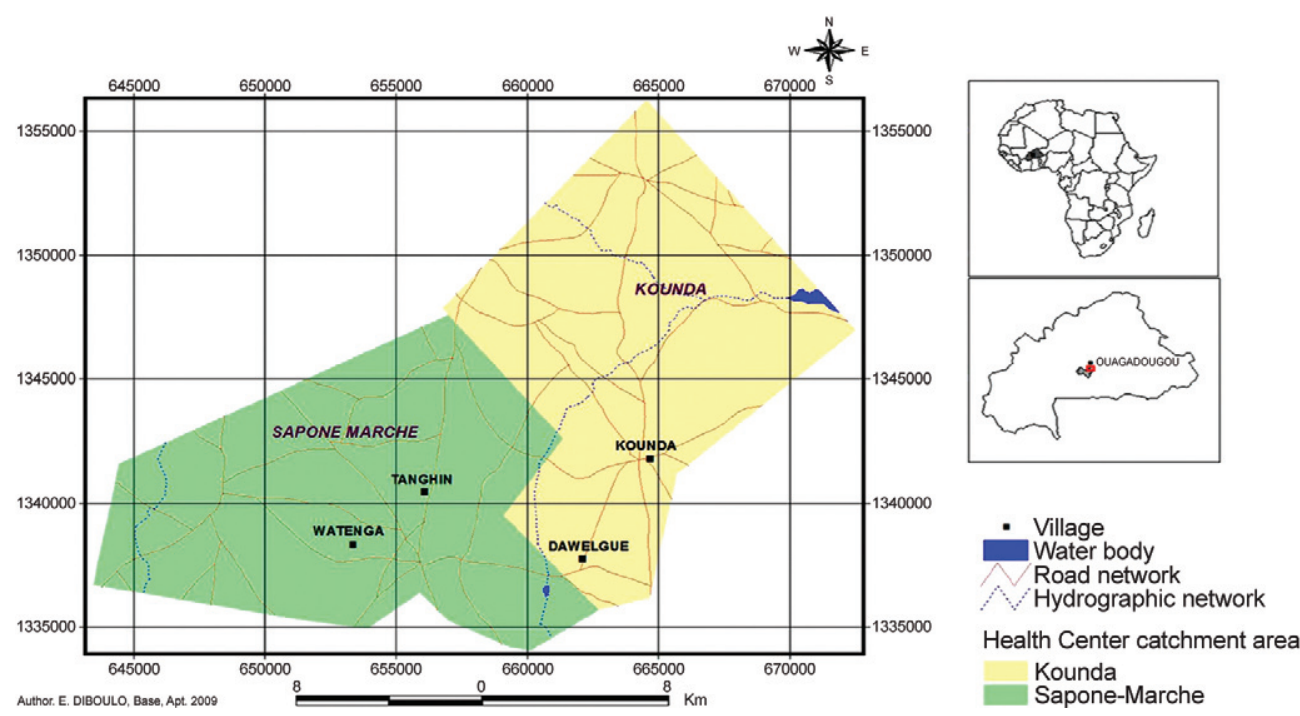

Fig. 1: study area. 
Blood films were stained with $15 \%$ Giemsa for 30 min. After drying at room temperature, the slides were independently examined using immersion microscopy by two blinded, experienced slide readers. The presence or absence of malaria parasites, Plasmodium species (P. falciparum, Plasmodium malariae and Plasmodium ovale), parasite stages (trophozoites, schizonts and gametocytes) and number of parasites per 200 leucocytes were determined by the readers. Slides were considered negative if no asexual parasite was found after the examination of 100 microscopy fields. The number of parasites per microlitre of blood was then calculated for each parasite stage and the results of both readers were averaged in the case of concordance between the two readers. The analysis of the concordance of the results (based on the species found, parasitaemia and parasite stage) was performed by a third party and any case of discrepancy was resolved by a third experienced microscopist using validated standard operating procedures.

Data entry and analysis - The data were entered and analysed using Epi Info 6.0 software (Centers for Disease Control and Prevention, Atlanta, GA, USA). The patients were categorised into two groups, a control group and an infected group, based on the microscopy results. The subjects with no detected malaria parasites were assigned to the control group and the subjects with malaria parasites were assigned to the infected group.

To test for any association between malaria parasitaemia (with P. falciparum) and a specific haematological parameter, we stratified $P$. falciparum parasitaemia into four categories (null, 1-1,000, 1,000-10,000 and > 10,000 parasites $/ \mu \mathrm{L}$ ) and calculated the mean $\mathrm{RBC}$, platelet, $\mathrm{Hb}$, lymphocyte, monocyte, basophil, eosinophil and basophil counts for each of these parasitaemia ranges. ANO$\mathrm{VA}$ and $t$ tests were used to compare the means of the haematological parameters for the two groups. A $p$ value of $\leq 0.05$ was considered to be statistically significant.

Ethics - The study was approved by the National Health Ethical Committee of Burkina Faso. Informed consent was obtained from the parents or guardians of all of the children participating prior to enrolment.

\section{RESULTS}

Of the 677 children less than five years of age whose parents or guardians allowed them to participate in the study, 469 children were screened by the study team, giving a survey response rate of $69.3 \%$. Of those screened, 434 apparently healthy children were enrolled in the study. In total, 35 children were excluded from the study because of acute disease confirmed by physicians. Of the children enrolled in the study, 20 were excluded from the analysis because only gametocytes were detected in their blood. Of the remaining 414 children, 222 were infected with asexual forms of malaria parasites and 192 children were free of malaria parasites. In the infected group, the parasite species infecting the children were as follows: $200(90.1 \%)$ children were infected with $P$. falciparum, one $(0.4 \%)$ child was infected with P. malariae, $15(6.6 \%)$ children had mixed infections with both P. falciparum and P. malariae and seven (1\%) children had mixed infections with both $P$. falciparum and $P$. ovale. The geometric mean of $P$. falciparum density was 2,786 (range of $1,917-3,264$ ) trophozoites $/ \mu \mathrm{L}$.

The mean age of the children in the asymptomatic Plasmodium-infected group was 41.8 months (range of 26.4-57.2) and the difference from the mean age of the children in the control group, 38.8 months (range of 22.455.2), was not statistically significant $(\mathrm{p}=0.06)$. The sex ratio $(\mathrm{M} / \mathrm{F})$ was 1.4 in the control group and 1.3 in the asymptomatic Plasmodium-infected group and this difference was not statistically significant $(\mathrm{p}=0.15)$.

The mean values of nine haematological parameters for the two groups are summarised in Table I.

The mean leucocyte count was lower in the infected group, but the difference was not statistically significant. However, the subsets of leucocytes were affected differently between the two groups. The mean lymphocyte count was significantly lower in the asymptomatic Plasmodium-infected group compared to the control

TABLE I

Mean value of haematological parameters in control and malaria infected groups

\begin{tabular}{|c|c|c|c|c|}
\hline Parameters $^{a}$ & & $\begin{array}{l}\text { Control group } \\
\quad(\mathrm{n}=192)\end{array}$ & $\begin{array}{l}\text { Infected group } \\
\quad(\mathrm{n}=222)\end{array}$ & $\mathrm{p}$ \\
\hline White blood cells & $\mathrm{x} 10^{3} / \mu \mathrm{L}$ & $9.622(3.262)$ & $9.520(3.297)$ & 0.75 \\
\hline Red blood cells & x $10^{3} / \mu \mathrm{L}$ & $4.388(536)$ & $4.158(563)$ & $<0.001$ \\
\hline Haemoglobin & $\mathrm{g} / \mathrm{dL}$ & $10.8(1.8)$ & $10.4(1.3)$ & $<0.001$ \\
\hline Platelets & x $10^{3} / \mu \mathrm{L}$ & $385(170)$ & $266(115)$ & $<0.0001$ \\
\hline Lymphocytes & $\times 10^{3} / \mu \mathrm{L}$ & $5.141(1.846)$ & 4.592 (1.999) & 0.004 \\
\hline Monocytes & x $10^{3} / \mu \mathrm{L}$ & $1.192(0.534)$ & $1.403(0.666)$ & $<0.001$ \\
\hline Neutrophils & x $10^{3} / \mu \mathrm{L}$ & $2.743(1.323)$ & $3.008(1.625)$ & 0.06 \\
\hline Eosinophils & x $10^{3} / \mu \mathrm{L}$ & $0.453(0.505)$ & $0.419(0.474)$ & 0.5 \\
\hline Basophils & x $10^{3} / \mu \mathrm{L}$ & $0.086(0.066)$ & $0.080(0.051)$ & 0.44 \\
\hline
\end{tabular}

$a$ : standard deviation. 
group ( $p<0.01)$, whereas the reverse was found for the monocyte count $(\mathrm{p}<0.001)$; no differences were observed for the neutrophil, eosinophil and basophil counts between the two groups. Stratifying the data by different $P$. falciparum parasitaemia ranges revealed a significant inverse relationship between the lymphocyte count and parasitaemia $(r=-0.17, p<0.001)$ and the eosinophil count and parasitaemia $(r=-0.15, \mathrm{p}<0.001)$. However, a significant positive correlation was found between the monocyte count and parasitaemia $(r=0.31, p<0.0001)$ and the neutrophil count and parasitaemia $(r=0.22$, $p$ $<0.001$ ), whereas no significant relationship was found between the basophil count and parasitaemia (Fig. 2), irrespective of age $(p=0.06)$ and $\operatorname{sex}(p=0.15)$.

The mean platelet count was significantly lower in the asymptomatic Plasmodium-infected group compared to the control group. Stratifying the data by different $P$. falciparum parasitaemia ranges, we found an inverse relationship between parasitaemia and the platelet count $(\mathrm{r}=-0.20, \mathrm{p}<0.001)$ (Fig. 2). Thrombocytopaenia, defined as a platelet count $<150 \times 10^{3} / \mu \mathrm{L}$, was reported in $31(14 \%)$ individuals in the asymptomatic Plasmodiuminfected group compared to seven $(3.6 \%)$ in the control group (Table II).

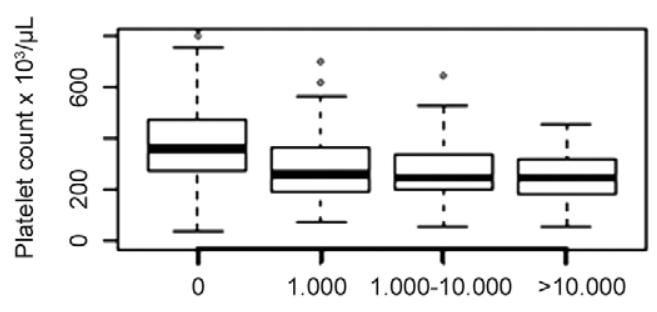

Plasmodium falciparum parasitaemia $(/ \mu \mathrm{L})$
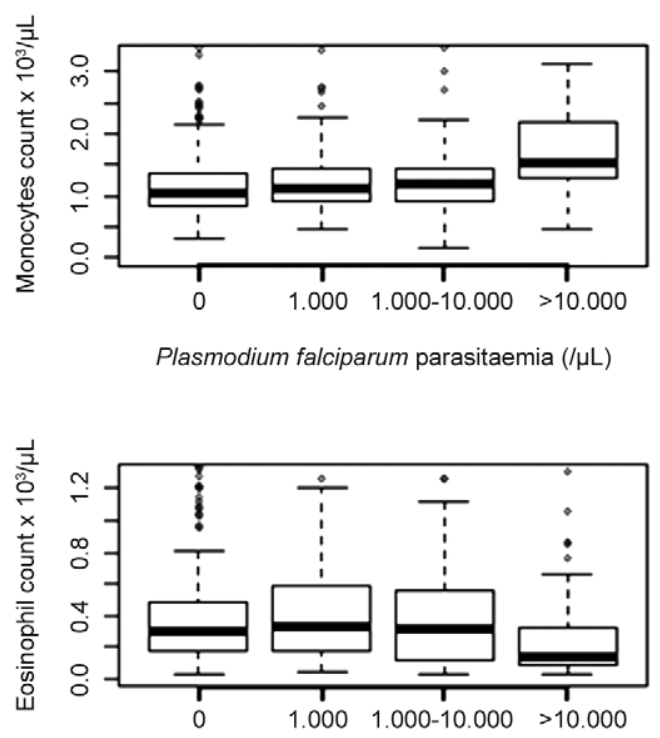

Plasmodium falciparum parasitaemia $(/ \mu \mathrm{L})$

\section{DISCUSSION}

The data presented here are part of a study evaluating mortality and morbidity related to malaria in which children were followed up for one year in the district of Sapone, which is a location where malaria transmission is stable and endemic. The cross-sectional survey was conducted at the peak of the malaria transmission season, a time when morbidity and mortality related to malaria are very high. In the rural health district of Sapone, we assumed that the socioeconomic and environmental conditions of the populations did not differ between the villages included in the study, correcting a selection bias between Plasmodium-infected and Plasmodiumuninfected children. Therefore, a socioeconomic and environmental gradient between the two study groups was unlikely to explain the study findings. This study confirms that haematological changes are frequent in Plasmodium infection, even if asymptomatic. Children with only gametocytes were excluded from the analysis because of the difficulty in classifying these children as infected or uninfected. Moreover, the goal of this study was to obtain certain information on the asymptomatic carriage of asexual Plasmodium infection and haematological parameters. However, an analysis of the

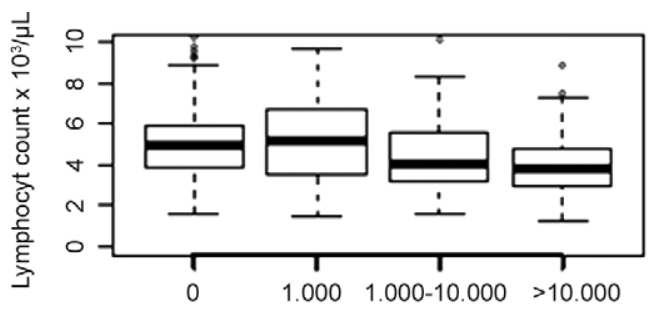

Plasmodium falciparum parasitaemia $(/ \mu \mathrm{L})$
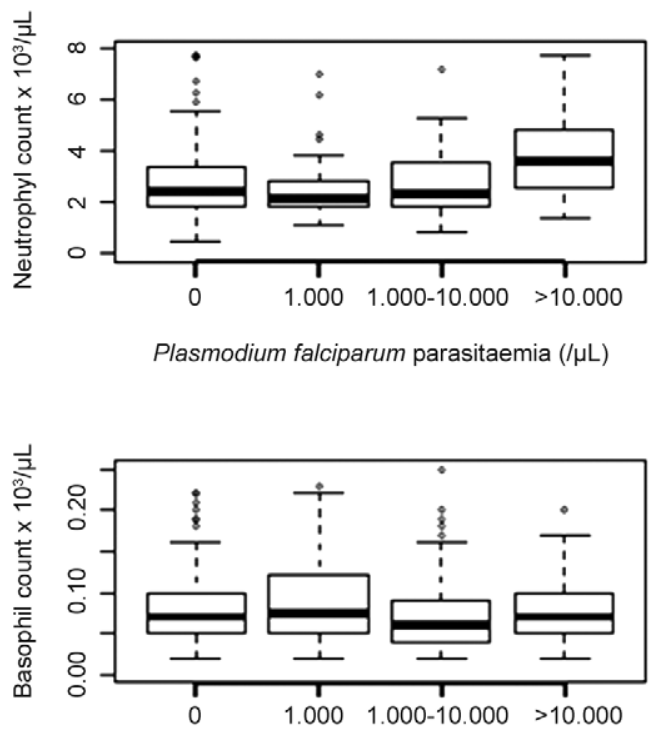

Plasmodium falciparum parasitaemia $(/ \mu \mathrm{L})$

Fig. 2: box and whisker plot showing median, interquartile and range platelet, lymphocyte, monocyte, neutrophil, eosinophil and basophil counts and proportions by range of parasitaemia. 
obtained data revealed no statistically significant differences between the asymptomatic infected children and uninfected children in terms of leucocyte, neutrophil, eosinophil or basophil count. Regarding the total leucocyte count, studies conducted in Ethiopia and Thailand have shown that adults with patent parasitaemia tend to have significantly lower WBC counts (Erhart et al. 2004, Kassa et al. 2006). The difference between these findings and ours could be explained by the age of the study participants (adults vs. children), confirming the view that Plasmodium infection pathogenesis is dependent on age. This result is corroborated by a study published by Maina et al. (2010), which reported the same finding for the leucocyte count in children from Kenya. Our results indicated that asymptomatic Plasmodium infection should not be assumed to explain observed changes in the total leucocyte, eosinophil or basophil count during vaccine clinical trials conducted in children living in our malaria-endemic area. However, asymptomatic parasitaemia of $>10,000$ parasites $/ \mu \mathrm{L}$ could increase the count of neutrophils. This finding was confirmed by Olliaro et al. (2011) in a study on a large sample of children less than five years of age with Plasmodium infection, showing that a high neutrophil count was consistently associated with higher parasitaemia.

However, the lymphocyte count was significantly lower in the children with asymptomatic Plasmodium infection, whereas the absolute monocyte count was higher compared to the count in the uninfected group. In a vaccine trial, efficacy is monitored through the assessment of immune responses to immunisations, requiring a high level of viable PBMCs after blood collection and handling. The significant decrease in the circulating lymphocyte count in the Plasmodium-infected group in the current study has also been observed in many published papers (Lisse et al. 1994, Richards et al. 1998, Erhart et al. 2004, Kassa et al. 2006). Apoptosis (ToureBalde et al. 1996, Helmby et al. 2000) and sequestration in lymphatic nodules (Langhorne \& Simon-Haarhaus 1991, Elhassan et al. 1994) are potential mechanisms explaining this decrease in circulating lymphocytes and these mechanisms of lymphocyte depletion are activated by Plasmodium infection. Similar findings were shown in a study conducted in Burkina Faso in which Sanou et al. (2012) demonstrated that individuals in the

\section{TABLE II}

Distribution of blood platelets count in control and malaria infected groups

\begin{tabular}{lcc}
\hline Platelet count & $\begin{array}{c}\text { Control group } \\
\mathrm{n}(\%)\end{array}$ & $\begin{array}{c}\text { Infected group }^{b} \\
\mathrm{n}(\%)\end{array}$ \\
\hline$<150 \times 10^{3} / \mu \mathrm{L}$ & $7(3.6)$ & $31(14)$ \\
$150-450 \times 10^{3} / \mu \mathrm{L}$ & $129(67.2)$ & $176(79.3)$ \\
$>450 \times 10^{3} / \mu \mathrm{L}$ & $56(29.2)$ & $15(6.7)$ \\
\hline
\end{tabular}

$a: \mathrm{n}=192 ; b: \mathrm{n}=222$. A significant difference in values $(\mathrm{p}<$ 0.01) was found between the two groups.
Fulani ethnic group, who are naturally less frequently infected by malaria parasites and with low parasitaemia compared to individuals in the Mossi ethnic group, had higher absolute lymphocyte counts than Mossi individuals in both malaria transmission seasons. Although there is a control group in vaccine trials, this finding must be considered in the assessment of immune responses to immunisations because a high number of lymphocytes cannot be obtained from asymptomatic Plasmodiuminfected children in comparison to uninfected children. Moreover, the validity of in vitro proliferative assays, such as the $\left[{ }^{3} \mathrm{H}\right]$ thymidine uptake assay, which is widely used in areas of endemicity to detect specific responses to P. falciparum antigens, could be affected. This situation could create a bias in vaccine trials in the assessment of immunogenicity if the number of asymptomatic Plasmodium-infected children in one group is higher than in the other group. In addition, asymptomatic Plasmodium-infected children, who occasionally have parasitaemia detectable only by molecular tools, may, at least theoretically, be erroneously considered to be negative controls in malaria field studies.

The assessment of subpopulations of lymphocytes $\left(\mathrm{CD}^{+}, \mathrm{CD}^{+}, \mathrm{CD}^{+}\right.$or other immunocompetent $\mathrm{T}$ cells) usually evaluated in vaccine trials must be investigated in infected children living in this malaria-endemic area. Moreover, co-infection with human immunodeficiency virus (HIV) could induce a bias in clinician judgement of people co-infected with HIV and malaria, as initiation, follow up and evaluation of antiretroviral treatment in HIV cases are dependent on the levels of subpopulations of $\mathrm{CD}^{+}$lymphocytes.

Although we observed a decrease in the lymphocyte count in asymptomatic Plasmodium-infected children, we did not find any difference in the leucocyte count between the two groups because the lower lymphocyte count in the infected children was compensated for by a significantly higher monocyte count. These monocytes also act as phagocytic, activated immune cells in the first line of defence against malaria parasite infection.

The Hb level, platelet count and RBC count were significantly lower in the children with Plasmodium infection, but the finding was not clinically significant.

According to international reference ranges, all the children in our study had mild anaemia: though the $\mathrm{Hb}$ level was significantly lower in the infected children, it was not clinically significant. The low $\mathrm{Hb}$ levels in blood samples from our study population are considered to be normal according to the reference ranges established in previous studies on African children between one-five years of age (Lugada et al. 2004, Quinto et al. 2006). Lugada et al. (2004) showed that the reference range for $\mathrm{Hb}$ in children less than five years of age in Uganda was $8.8-12.5 \mathrm{~g} / \mathrm{dL}$ and Quinto reported $6.5-13.5 \mathrm{~g} / \mathrm{dL}$ as the reference in southern Mozambique. A low Hb level is frequently associated with Plasmodium infection in children and is caused by the destruction of infected RBCs and/or a decrease in cell production (Phillips \& Pasvol 1992). In sub-Saharan Africa, many other factors contribute to decreased $\mathrm{Hb}$ levels in children, includ- 
ing poor nutritional status, soil-transmitted helminths or other intestinal parasite infections and poor access to health care. According to a WHO (2004) report, it is important to evaluate anaemia in two instances in a malaria vaccine trial: after the end of the rainy season and at the start of the rainy season. A study conducted in Ghana reported that $24 \%$ of children aged 12-24 months suffered from severe anaemia after the end of the rainy season, whereas only $2 \%$ of them suffered from anaemia at the start of the rainy season, suggesting that anaemia could be a good biological marker (WHO 2004). In addition to Plasmodium infection, others parameters should be considered when interpreting anaemia in children living in malaria-endemic areas and involved in vaccine trials.

The blood platelet count was significantly reduced in the asymptomatic infected children in this study. Only platelet counts were stratified in this analysis because several studies have already shown an inverse correlation between $P$. falciparum parasitaemia in clinical malaria and blood platelet counts (Ladhani et al. 2002, Erhart et al. 2004, Casals-Pascual et al. 2006). Thrombocytopaenia was inversely related to parasite density and decreased with increasing levels of $P$. falciparum parasitaemia. Thus, blood platelets appear to be a good, but nonspecific marker of malaria outcome in children, a finding that has already been reported (Ladhani et al. 2002, Erhart et al. 2004, Casals-Pascual et al. 2006). The decrease in blood platelets in asymptomatic Plasmodi$u m$-infected children may be explained by peripheral cell destruction and consumption and also by a reduction in cell production. Therefore, the interpretation of thrombocytopaenia in vaccine trials in children living in malaria-endemic areas should consider the presence or absence of $P$. falciparum parasitaemia.

All children with the asymptomatic carriage of Plasmodium were treated according to the national malaria control programme guidelines, but we did not investigate whether the children's haematological values posttreatment became similar to the parameters of the control group.

These findings suggest that malaria parasites may affect the haematopoiesis of children living in malaria-endemic areas. However, the decreased levels of lymphocytes and $\mathrm{Hb}$ might be affected by peripheral destruction. Special attention should be applied when interpreting haematological parameters, such as RBC, Hb, platelet, lymphocyte and monocyte levels and when evaluating immune responses in children living in endemic areas and enrolled in vaccine trials. The assessment of subpopulations of lymphocytes (e.g., $\mathrm{CD} 4^{+}, \mathrm{CD} 8^{+}, \mathrm{CD}^{+}$, natural killer) usually performed in vaccine trials must be investigated and local laboratory reference ranges should be established to accurately evaluate the changes in biological parameters in clinical trials.

\section{ACKNOWLEDGEMENTS}

To the population of Dawelgue, Tanghin, Kounda and Watenga and to the Sapone District Health Officers and staff, for cooperation and support to the study, and to Bianca Zingales, for reviewing the manuscript.

\section{REFERENCES}

Amodu OK, Adeyemo AA, Olumese PE, Gbadegesin RA 1998. Intraleucocytic malaria pigment and clinical severity of malaria in children. Trans R Soc Trop Med Hyg 92: 54-56.

Casals-Pascual C, Kai O, Newton CR, Peshu N, Roberts DJ 2006. Thrombocytopenia in falciparum malaria is associated with high concentrations of IL-10. Am J Trop Med Hyg 75: 434-436.

DEP/SNIS - Direction des Etudes et de la Planification/Service de l'information Sanitaire 2007. Annuaire Statistique/Sante 2006, Ministere de La Sante/Secretaria General/DEP, Burkina Faso, $243 \mathrm{pp}$.

Elhassan IM, Hviid L, Satti G, Akerstrom B, Jakobsen PH, Jensen JB, Theander TG 1994. Evidence of endothelial inflammation, T cell activation and $\mathrm{T}$ cell reallocation in uncomplicated Plasmodium falciparum malaria. Am J Trop Med Hyg 51: 372-379.

Erhart LM, Yingyuen K, Chuanak N, Buathong N, Laoboonchai A, Miller RS, Meshnick SR, Gasser Jr RA, Wongsrichanalai C 2004. Hematologic and clinical indices of malaria in a semi-immune population of western Thailand. Am J Trop Med Hyg 70: 8-14.

Fisher GU, Gordon MP, Lobel HO, Runcik K 1970. Malaria in soldiers returning from Vietnam. Epidemiologic, therapeutic and clinical studies. Am J Trop Med Hyg 19: 27-39.

Helmby H, Jonsson G, Troye-Blomberg M 2000. Cellular changes and apoptosis in the spleens and peripheral blood of mice infected with blood-stage Plasmodium chabaudi chabaudi AS. Infect Immun 68: 1485-1490.

Hill AV, Allsopp CE, Kwiatkowski D, Anstey NM, Twumasi P, Rowe PA, Bennett S, Brewster D, McMichael AJ, Greenwood BM 1991. Common west African HLA antigens are associated with protection from severe malaria. Nature 352: 595-600.

Kassa D, Petros B, Mesele T, Hailu E, Wolday D 2006. Characterization of peripheral blood lymphocyte subsets in patients with acute Plasmodium falciparum and $P$. vivax malaria infections at Wonji Sugar Estate, Ethiopia. Clin Vaccine Immunol 13: 376-379.

Ladhani S, Lowe B, Cole AO, Kowuondo K, Newton CR 2002. Changes in white blood cells and platelets in children with falciparum malaria: relationship to disease outcome. Br J Haematol 119: 839-847.

Langhorne J, Simon-Haarhaus B 1991. Differential T cell responses to Plasmodium chabaudi chabaudi in peripheral blood and spleens of C57BL/6 mice during infection. J Immunol 146: 2771-2775.

Lisse IM, Aaby P, Whittle H, Knudsen K 1994. A community study of T lymphocyte subsets and malaria parasitaemia. Trans $R$ Soc Trop Med Hyg 88: 709-710.

Lugada ES, Mermin J, Kaharuza F, Ulvestad E, Were W, Langeland N, Asjo B, Malamba S, Downing R 2004. Population-based hematologic and immunologic reference values for a healthy Ugandan population. Clin Diagn Lab Immunol 11: 29-34.

Maina RN, Walsh D, Gaddy C, Hongo G, Waitumbi J, Otieno L, Jones D, Ogutu BR 2010. Impact of Plasmodium falciparum infection on haematological parameters in children living in Western Kenya. Malar J 9 (Suppl. 3): S4.

Olliaro P, Djimde A, Dorsey G, Karema C, Martensson A, Ndiaye JL, Sirima SB, Vaillant M, Zwang J 2011. Hematologic parameters in pediatric uncomplicated Plasmodium falciparum malaria in subSaharan Africa. Am J Trop Med Hyg 85: 619-625.

Perera MK, Carter R, Goonewardene R, Mendis KN 1994. Transient increase in circulating gamma/delta $\mathrm{T}$ cells during Plasmodium vivax malarial paroxysms. J Exp Med 179: 311-315.

Phillips RE, Pasvol G 1992. Anaemia of Plasmodium falciparum malaria. Baillieres Clin Haematol 5: 315-330. 
Quinto L, Aponte JJ, Sacarlal J, Espasa M, Aide P, Mandomando I, Guinovart C, Macete E, Navia MM, Thompson R, Menendez C, Alonso PL 2006. Haematological and biochemical indices in young African children: in search of reference intervals. Trop Med Int Health 11: 1741-1748.

Richards MW, Behrens RH, Doherty JF 1998. Hematologic changes in acute, imported Plasmodium falciparum malaria. Am J Trop Med Hyg 59: 859.

Rowe AK, Rowe SY, Snow RW, Korenromp EL, Schellenberg JR, Stein C, Nahlen BL, Bryce J, Black RE, Steketee RW 2006. The burden of malaria mortality among African children in the year 2000. Int J Epidemiol 35: 691-704.

Sanou GS, Tiendrebeogo RW, Ouedraogo AL, Diarra A, Ouedraogo A, Yaro JB, Ouedraogo E, Verra F, Behr C, Troye-Blomberg M, Modiano D, Dolo A, Torcia MG, Traore Y, Sirima SB, Nebie I 2012. Haematological parameters, natural regulatory $\mathrm{CD}^{+}$ $\mathrm{CD} 25^{+} \mathrm{FOXP}^{+} \mathrm{T}$ cells and gammadelta $\mathrm{T}$ cells among two sympatric ethnic groups having different susceptibility to malaria in Burkina Faso. BMC Res Notes 5: 76.
Schellenberg D, Menendez C, Kahigwa E, Font F, Galindo C, Acosta C, Schellenberg JA, Aponte JJ, Kimario J, Urassa H, Mshinda H, Tanner M, Alonso P 1999. African children with malaria in an area of intense Plasmodium falciparum transmission: features on admission to the hospital and risk factors for death. Am J Trop Med Hyg 61: 431-438.

Snow RW, Craig M, Deichmann U, Marsh K 1999. Estimating mortality, morbidity and disability due to malaria among Africa's nonpregnant population. Bull World Health Organ 77: 624-640.

Stein CM, Gelfand M 1985. The clinical features and laboratory findings in acute Plasmodium falciparum malaria in Harare, Zimbabwe. Cent Afr J Med 31: 166-170.

Toure-Balde A, Sarthou JL, Aribot G, Michel P, Trape JF, Rogier C, Roussilhon C 1996. Plasmodim falciparum induces apoptosis in human mononuclear cells. Infect Immun 64: 744-750.

WHO - World Health Organization 2004. Evaluation of malaria vaccines: end-points and trial design of phase $2 b$ clinical trails of blood-stage vaccines, WHO, Geneva, $58 \mathrm{pp}$. 